\title{
First, do no harm: bilateral radial artery occlusion in a COVID-19 patient
}

\author{
Anne Bouwhuis ${ }^{1,2} \cdot$ Patrick Schober $^{2} \cdot$ Leo M. A. Heunks $^{1} \cdot$ Lothar A. Schwarte $^{2}$ (I)
}

Received: 8 June 2020 / Accepted: 30 June 2020 / Published online: 6 July 2020

(c) Springer Nature B.V. 2020

\begin{abstract}
Objective Objective of this case report is to draw attention to a less known thrombotic complication associated with COVID19, i.e., thrombosis of both radial arteries, with possible (long-term) consequences.

The Case In our COVID-19 ICU a 49-year-old male patient was admitted, with past medical history of obesity, smoking and diabetes, but no reported atherosclerotic complications. The patient had been admitted with severe hypoxemia and multiple pulmonary emboli were CT-confirmed. ICU-treatment included mechanical ventilation and therapeutic anticoagulation. Preparing the insertion of a new radial artery catheter for invasive blood pressure measurement and blood sampling, we detected that both radial arteries were non-pulsating and occluded: (a) Sonography showed the typical anatomical localization of both radial and ulnar arteries. However, Doppler-derived flow-signals could only be obtained from the ulnar arteries. (b) To test collateral arterial supply of the hand, a pulse-oximeter was placed on the index finger. Thereafter, the ulnar artery at the wrist was compressed. This compression caused an immediate loss of the finger's pulse-oximetry perfusion signal. The effect was reversible upon release of the ulnar artery. (c) To test for collateral perfusion undetectable by pulse-oximetry, we measured regional oxygen saturation $\left(\mathrm{rSO}_{2}\right)$ of the thenar muscle by near-infrared spectroscopy (NIRS). Confirming our findings above, ulnar arterial compression demonstrated that thenar $\mathrm{rSO}_{2}$ was dependent on ulnar artery flow. The described development of bilateral radial artery occlusion in a relatively young and therapeutically anticoagulated patient with no history of atherosclerosis was unexpected.

Conclusions Since COVID-19 patients are at increased risk for arterial occlusion, it appears advisable to meticulously check for adequacy of collateral (hand-) perfusion, avoiding the harm of hand ischemia if interventions (e.g., catheterizations) at the radial or ulnar artery are intended.
\end{abstract}

Keywords COVID-19 $\cdot$ Radial $\cdot$ Artery $\cdot$ Occlusion $\cdot$ Thrombosis

COVID-19 has originally been described as respiratory syndrome, but cardiovascular complications are increasingly recognized. COVID-19 related cardiovascular complications include venous thrombosis with pulmonary emboli [1], and primary arterial occlusions [2]. In clinical focus are the acutely life-threatening cardiovascular complications, e.g., pulmonary vessel thrombosis, or cerebral and coronary occlusions [3]. Here, in addition, we would like

Lothar A. Schwarte

L.Schwarte@AmsterdamUMC.NL

1 Department of Intensive Care, Amsterdam University Medical Center (Location VUMC), Amsterdam, Netherlands

2 Department of Anesthesiology, Amsterdam University Medical Center (Location VUMC), De Boelelaan 1117, 1081HV Amsterdam, Netherlands to draw attention to another thrombotic complication with possible (long-term) consequences, i.e., thrombosis of the radial artery.

\section{The Case}

In our COVID-19 ICU a 49-year-old male patient was admitted, with past medical history of obesity, smoking and diabetes, but no reported atherosclerotic complications. The patient had been admitted with severe hypoxemia and multiple pulmonary emboli were CT-confirmed. ICUtreatment included mechanical ventilation and therapeutic anticoagulation.

Preparing the insertion of a new radial artery catheter (ICU-day 29) for invasive blood pressure measurement and 

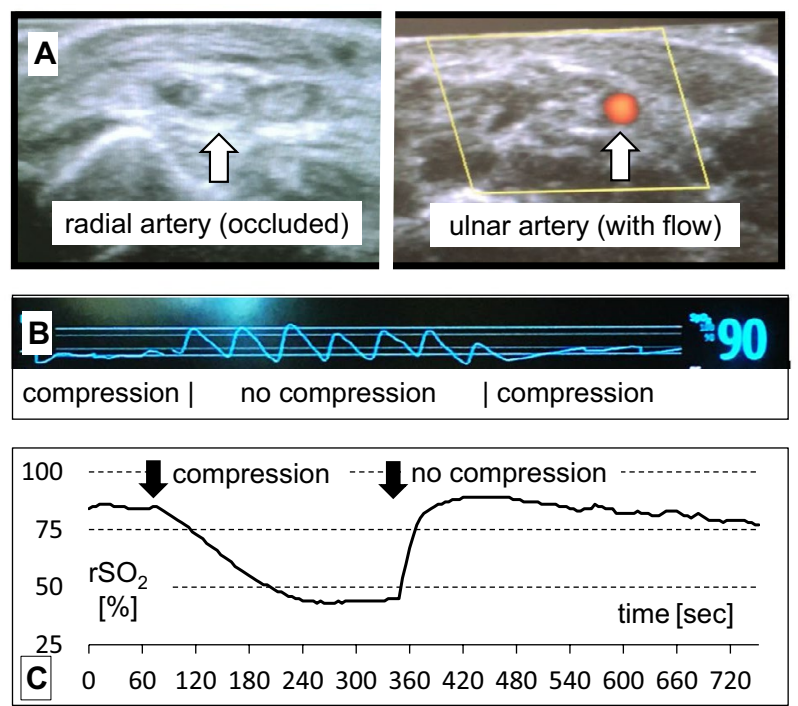

Fig. 1 a-c Doppler flow signals were absent in both radial arteries, but present in both ulnar arteries (a). In line with this, fingerclip pulse-oximetry waveforms were lost after compression of an ulnar artery (b) confirming dependency of the hand's perfusion on the ulnar artery. This is also confirmed by impaired regional thenar muscle oxygen saturation $\left(\mathrm{rSO}_{2}\right.$, measured by near-infrared spectroscopy, NIRS, Nonin-SenSmart ${ }^{\circledR}$ ) after selective ulnar artery compression (c): In the described COVID-19 patient, selective compression of the right ulnar artery (starting at $\sim 60 \mathrm{~s}$ ) induced a marked drop in NIRS-measured $\mathrm{rSO}_{2}$, leveling off after 3 min of ischemia (starting at $\sim 240 \mathrm{~s}$ ). After reaching stable, low values, the compression of the ulnar artery was released (at $\sim 340 \mathrm{~s}$ ), resulting in a rapid recovery of $\mathrm{rSO}_{2}$ and return to baseline thenar $\mathrm{rSO}_{2}$

blood sampling, we detected that both radial arteries were non-pulsating and occluded:

- Sonography showed the typical anatomical localization of both radial and ulnar arteries. However, Dopplerderived flow-signals could only be obtained from the ulnar arteries (Fig. 1a).

- To test collateral arterial supply of the hand, a pulseoximeter was placed on the index finger. Thereafter, the ulnar artery at the wrist was compressed [4]. This compression caused an immediate loss of the finger's pulseoximetry perfusion signal. The effect was reversible upon release of the ulnar artery (Fig. 1b).

- To test for collateral perfusion undetectable by pulse-oximetry, we measured regional oxygen saturation $\left(\mathrm{rSO}_{2}\right)$ of the thenar muscle by near-infrared spectroscopy (NIRS) [5]. Confirming our findings above, ulnar arterial compression demonstrated that thenar $\mathrm{rSO}_{2}$ was dependent on ulnar artery flow (Fig. 1c).

Venous thrombotic complications are not uncommon in COVID-19 patients [1]. However, the described development of bilateral radial artery occlusion in a relatively young and therapeutically anticoagulated patient with no history of atherosclerosis was unexpected. The etiology of these bilateral radial artery occlusions is probably multifactorial, including vascular lesions from previous catheter placements, but likely also include a COVID-19 related hypercoagulatory state.

Given the limited long-term experience with COVID-19 patients so far, it is unclear if those arterial occlusions will resolve spontaneously, or create long term sequelae, e.g., unavailability of the radial artery for angiography access or unavailability of the radial artery for coronary-bypass grafting in the future.

Since COVID-19 patients are at increased risk for arterial occlusion, it appears advisable to meticulously check for adequacy of collateral (hand-) perfusion [4], avoiding the harm of hand ischemia if interventions (e.g., catheterizations) at the radial or ulnar artery are intended.

Author contributions $\mathrm{AB}$ : manuscript, figures, follow-up. PS: manuscript. LMAH: manuscript. LAS: concept, manuscript, figures. All authors read and approved the final manuscript version.

Funding No funding.

Data availability Yes, recorded data are available from the corresponding author.

\section{Compliance with ethical standards}

Conflict of interest The authors have no conflicts of interest.

Ethical approval Not applicable.

Consent to participate Not applicable.

Consent for publication Not applicable.

\section{References}

1. Levi M, Thachil J, Iba T, Levy JH. Coagulation abnormalities and thrombosis in patients with COVID-19. The Lancet Haematology. 2020. https://doi.org/10.1016/S2352-3026(20)30145-9.

2. Perini P, Nabulsi B, Massoni CB, Azzarone M, Freyrie A. Acute limb ischaemia in two young, non-atherosclerotic patients with COVID-19. The Lancet. 2020;395:1546.

3. Bikdeli B, Madhavan MV, Jimenez D, et al. COVID-19 and thrombotic or thromboembolic disease: implications for prevention, antithrombotic therapy, and follow-up. J Am Coll Cardiol. 2020. https://doi.org/10.1016/j.jacc.2020.04.031.

4. Vukovic P, Peric M, Radak S, et al. Preoperative insight into the quality of radial artery grafts. Angiology. 2017;68:790-4.

5. Scheeren TWL, Schober P, Schwarte LA. Monitoring tissue oxygenation by near infrared spectroscopy (NIRS): background and current applications. J Clin Monit Comput. 2012;26:279-87.

Publisher's Note Springer Nature remains neutral with regard to jurisdictional claims in published maps and institutional affiliations. 